Pacific

Journal of

Mathematics

\title{
COMPLEX INTERPOLATION AND TWISTED TWISTED HILBERT SPACES
}

Félix Cabello Sánchez, Jesús M. F. Castillo and Nigel J. Kalton 


\title{
COMPLEX INTERPOLATION AND TWISTED TWISTED HILBERT SPACES
}

\author{
Félix Cabello SÁnchez, Jesús M. F. Castillo and Nigel J. Kalton
}

\begin{abstract}
We show that Rochberg's generalized interpolation spaces $\mathscr{X}^{(n)}$ arising from analytic families of Banach spaces form exact sequences $\mathbf{0} \rightarrow \mathscr{X}^{(n)} \rightarrow$ $\mathscr{L}^{(n+k)} \rightarrow \mathscr{X}^{(k)} \rightarrow \mathbf{0}$. We study some structural properties of those sequences; in particular, we show that nontriviality, having strictly singular quotient map, or having strictly cosingular embedding depend only on the basic case $n=k=1$. If we focus on the case of Hilbert spaces obtained from the interpolation scale of $\ell_{p}$ spaces, then $\mathscr{L}^{(2)}$ becomes the well-known KaltonPeck space $Z_{2}$; we then show that $\mathscr{L}^{(n)}$ is (or embeds in, or is a quotient of) a twisted Hilbert space only if $n=1,2$, which solves a problem posed by David Yost; and that it does not contain $\ell_{2}$ complemented unless $n=1$. We construct another nontrivial twisted sum of $Z_{2}$ with itself that contains $\ell_{2}$ complemented.
\end{abstract}

\section{Introduction}

Kalton and Peck [1979, Section 4] developed a method to produce nontrivial selfextensions of most quasi-Banach spaces with unconditional basis, including all Banach spaces apart from $c_{0}$; see [Cabello Sánchez et al. 2012, Theorem 1]. The most shining examples are perhaps the so-called $Z_{p}$ spaces, which are twisted sums of the $\ell_{p}$ spaces. If, however, one wants to construct twisted sums of $Z_{p}$, the Kalton-Peck method simply does not work because of their poor unconditional structure. On the other hand, the existence of such twisted sums is guaranteed by the local theory of exact sequences, at least when $p>1$; see, e.g., [Cabello Sánchez and Castillo 2004]. Our starting goal with this paper was to develop a method to obtain twisted sums of twisted sum spaces, keeping the $Z_{p}$ spaces as the control case.

The path connecting interpolation theory and twisted sums was opened by Rochberg and Weiss, who introduce [1983] certain spaces which naturally arise in the study of "analytic families" of Banach spaces and that turn out to be twisted sums of the "intermediate" spaces. Actually, if $\mathscr{F}$ is the usual Calderón space of analytic

This research has been supported in part by project MTM2013-45643-C2-1-P.

MSC2010: 46B20, 46B70, 46M18, 46M35.

Keywords: Complex interpolation, twisted sums of Banach spaces. 
functions on the strip $0<\Re z<1$ associated to the couple $\left(\ell_{\infty}, \ell_{1}\right)$ in the complex interpolation method, then, as is well known, $\left[\ell_{\infty}, \ell_{1}\right]_{\theta}=\{f(\theta): f \in \mathscr{F}\}=\ell_{p}$, where $p=1 / \theta$ for $0<\theta<1$ and $Z_{p}$ is isomorphic to

$$
\left\{\left(f^{\prime}(\theta), f(\theta)\right): f \in \mathscr{F}\right\},
$$

with the quotient norm inherited from $\mathscr{F}$ — though this is not made explicit in [loc. cit.].

Nothing seems to prevent one from adding more derivatives to (1) and figuring out that the resulting space represents the iterated twisted sum spaces. Rochberg [1996] did just this, in the broader setting of analytic families of Banach spaces. Accomplishing that is not, by far, as simple as it sounds; and perhaps the turning point in Rochberg's approach is the use of Taylor coefficients instead of merely derivatives, as is suggested in [Kalton and Montgomery-Smith 2003, Section 10, p. 1161].

In this paper, we adopt such an approach, which can be considered a spin-off from that of Rochberg [1996], with several variations, the first of which is the use of admissible spaces of analytic functions instead of analytic families, which makes "reiteration" both unavailable and unnecessary. Thus, given an admissible space of analytic functions $\mathscr{F}_{F}$, we consider the space $\mathscr{X}^{(n)}$ of all possible lists of length $n$ of Taylor coefficients of functions in $\mathscr{F}_{F}$ - at a fixed point $z$, which is understood from now on - endowed with the obvious infimum norm on it. Then we observe that if $m=n+k$, there is an exact sequence

$$
0 \longrightarrow \mathscr{L}^{(n)} \longrightarrow \mathscr{L}^{(m)} \longrightarrow \mathscr{L}^{(k)} \longrightarrow 0
$$

and so $\mathscr{X}^{(m)}$ is a twisted sum of $\mathscr{X}^{(n)}$ and $\mathscr{X}^{(k)}$. The key nontrivial step here is obtaining the right form of the embedding. To this we devote Section 3 in which we obtain two (equivalent) representations for the embedding, depending on the representation of the spaces. Regarding the sequences themselves, we will show that many properties - such as nontriviality, having strictly singular quotient map, or having strictly cosingular embedding — depend only on the seed case $n=k=1$. The nontriviality of this case has to be worked apart.

We then focus on the case in which $\mathscr{F}$ is the Calderón space of the couple $\left(\ell_{\infty}, \ell_{1}\right)$. If we fix $z=\frac{1}{2}$, then $\mathscr{X}^{(1)}=\ell_{2}$ and $\mathscr{X}^{(2)}$ is the Kalton-Peck space $Z_{2}$ [1979]. The space $\mathscr{L}^{(3)}$ is both a twisted sum of $\ell_{2}$ with $Z_{2}$ and a twisted sum of $Z_{2}$ with $\ell_{2}$, and $\mathscr{X}^{(4)}$ is, among other possibilities, a twisted sum of $Z_{2}$ with itself, as desired. We then pass to establish structural properties of the spaces $\mathscr{X}^{(n)}$ and of the sequences (2). Regarding the spaces, we will show that $\mathscr{L}^{(n)}$ is (or embeds in, or is a quotient of) a twisted Hilbert space only if $n=1,2-$ which solves a problem posed by David Yost — and that it does not contain $\ell_{2}$ complemented unless $n=1$. To put this result in perspective, we will construct a nontrivial twisted sum of $Z_{2}$ with itself that contains $\ell_{2}$ complemented. 


\section{Preliminaries}

We warmly recommend the reader who is not familiar with [Kalton and Peck 1979] or [Rochberg 1996] to postpone reading this article until getting acquainted with them. Perusing the papers [Cwikel et al. 1989; Rochberg 2007], the article [Kalton and Montgomery-Smith 2003], and the monograph [Castillo and González 1997] can help with the background. Anyway, the basic ingredients to read this paper are described next.

2.1. Exact sequences. A short sequence of Banach spaces and (linear, bounded) operators

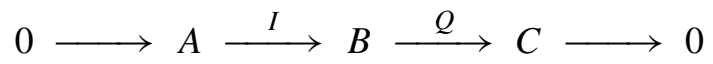

is said to be exact if the kernel of each arrow equals the image of the preceding one. As $I(A)$ is closed in $B$ the operator $I$ embeds $A$ as a subspace of $B$ and $C$ is isomorphic to the quotient $B / I(A)$, by the open mapping theorem. For this reason one often says that $B$ is a twisted sum of $A$ with $C$ (in that order); the whole sequence (3) is said to be an extension of $C$ by $A$ (the order was reversed for "functorial" reasons).

The extension (3) is said to be trivial if there is an operator $P: B \rightarrow A$ such that $P \circ I=\mathbf{1}_{A}$, i.e., $I(A)$ is complemented in $B$; equivalently, there is an operator $J: C \rightarrow B$ such that $Q \circ J=\mathbf{1}_{C}$. In this case $P \times Q: B \rightarrow A \times C$ is an isomorphism, with inverse $I \oplus J$, and thus the "twisted sum" $B$ is (isomorphic to) the direct sum $A \oplus C=A \times C$.

2.2. Admissible spaces of analytic functions. We will work within the framework of an admissible space of analytic functions as defined in [Kalton and MontgomerySmith 2003, Section 10]. So, let $U$ be an open set of $\mathbb{C}$, conformally equivalent to the disc $\mathbb{D}=\{z \in \mathbb{C}:|z|<1\}$, and let $W$ be a complex Banach space. A Banach space $\mathscr{F}$ of analytic functions $F: U \rightarrow W$ is said to be admissible provided:

(a) For each $z \in U$, the evaluation map $\delta_{z}: \mathscr{F} \rightarrow W$ is bounded.

(b) If $\varphi: U \rightarrow \mathbb{D}$ is a conformal equivalence, then $F \in \mathscr{F}$ if and only if $\varphi \cdot F \in \mathscr{F}$ and $\|\varphi \cdot F\|_{\mathscr{F}}=\|F\|_{\mathscr{F}}$.

For each $z \in U$ we define $X_{z}=\{x \in W: x=F(z)$ for some $F \in \mathscr{F}\}$ with the norm $\|x\|=\inf \left\{\|F\|_{\mathscr{F}}: x=F(z)\right\}$ so that $X_{z}$ is isometric to $\mathscr{F} / \operatorname{ker} \delta_{z}$ with the quotient norm. One often says that $\left(X_{z}\right)_{z \in U}$ is an analytic family of Banach spaces. The simplest examples arise from complex interpolation. Indeed, let $\left(X_{0}, X_{1}\right)$ be a Banach couple and take $W=X_{0}+X_{1}$ and $U$ the strip $0<\Re z<1$. Let $\mathscr{F}=\mathscr{C}\left(X_{0}, X_{1}\right)$ be the Calderón space of those continuous functions $F: \bar{U} \rightarrow W$ which are analytic on $U$ and satisfy the boundary conditions: for $k=0,1$, one has 
$F(k+t i) \in X_{k}$ and $\|F\|_{\mathscr{C}}=\sup \left\{\|F(k+t i)\|_{X_{k}}: t \in \mathbb{R}, k=0,1\right\}<\infty$. Then $\mathscr{F}_{\mathcal{F}}$ is admissible and $X_{z}=\left[X_{0}, X_{1}\right]_{\theta}$, with $\theta=\Re z$, is an analytic family.

It is important now to realize that when $\mathscr{F}_{F}$ is admissible, the map $\delta_{z}^{n}: \mathscr{F} \rightarrow W$, evaluation of the $n$-th derivative at $z$, is bounded for all $z \in U$ and all $n \in \mathbb{N}$ by an iterated use of (a), the definition of derivative, and the principle of uniform boundedness. Thus, it makes sense to consider the Banach spaces

$$
\mathscr{F} / \bigcap_{i<n} \operatorname{ker} \delta_{z}^{i} \quad \text { for } n \in \mathbb{N} \text {. }
$$

\section{Exact sequences of derived spaces}

3.1. Lists of Taylor coefficients. Following Rochberg, let us fix $z \in U$ and consider the following spaces:

$$
\mathscr{X}_{z}^{(n)}=\left\{\left(x_{n-1}, \ldots, x_{0}\right) \in W^{n}: x_{i}=\hat{f}[i ; z] \text { for some } f \in \mathscr{F} \text { and all } 0 \leq i<n\right\},
$$

where $\hat{f}[i ; z]=f^{(i)}(z) / i$ ! is the $i$-th Taylor coefficient of $f$ at $z$. Thus, the elements of $\mathscr{L}_{z}^{(n)}$ are (truncated) sequences of Taylor's coefficients (at $z$ ) of functions in $\mathscr{F}$ arranged in decreasing order. Here, we deviate from Rochberg notation in two points: first, the superscript $(n)$ refers to the "number of variables" and not to the highest derivative, and second, we have arranged Taylor coefficients decreasingly in order to match with the usual notation for twisted sums, with the subspace on the left and the quotient on the right. If we equip $\mathscr{X}_{z}^{(n)}$ with the obvious quotient norm, then it is isometric to $\mathscr{F} / \bigcap_{i<n} \operatorname{ker} \delta_{z}^{i}$ via Taylor coefficients and so it is complete. From now on we shall omit the base point $z$, which is understood to be fixed.

3.2. Operators. Next, we introduce certain "natural" operators linking the various spaces $\mathscr{L}^{(n)}$ as $n$ varies. Those operators will be used to construct the exact sequences we want.

To this end, for $1 \leq n, k<m$ we denote by $\imath_{n, m}: W^{n} \rightarrow W^{m}$ the inclusion on the left given by $\imath_{n, m}\left(x_{n}, \ldots, x_{1}\right)=\left(x_{n}, \ldots, x_{1}, 0 \ldots, 0\right)$ and by $\pi_{m, k}: W^{m} \rightarrow W^{k}$ the projection on the right given by $\pi_{m, k}\left(x_{m}, \ldots, x_{k}, \ldots, x_{1}\right)=\left(x_{k}, \ldots, x_{1}\right)$. While $\pi_{m, k}$ is obviously a quotient map from $\mathscr{L}^{(m)}$ onto $\mathscr{L}^{(k)}$, it is not clear at all that $\boldsymbol{l}_{n, m}$ maps $\mathscr{L}^{(k)}$ to $\mathscr{L}^{(n)}$, let alone that it is continuous. To prove that this is indeed the case, we need to do some extra work.

Observe that if $\varphi$ is as in (b) and if $\Pi$ is a "polynomial" in $\varphi$; that is, $\Pi=\sum_{i} a_{i} \varphi^{i}$ for some finite sequence of complex numbers $\left(a_{i}\right)$, then $\Pi \cdot f \in \mathscr{F}_{\text {for each } f \in \mathscr{F}}$ and $\|\Pi \cdot f\|_{\mathscr{F}} \leq\left(\sum_{i}\left|a_{i}\right|\right)\|f\|_{\mathscr{F}}$.

Lemma 1. Let $\varphi: U \rightarrow \mathbb{D}$ be a conformal equivalence vanishing at $z$. Then, for $0 \leq k \leq m$, there is a polynomial $P$ of degree at most $m$ such that

$$
\widehat{P \circ \varphi}[i ; z]=\delta_{i k} \quad \text { for every } 0 \leq i \leq m .
$$


Proof. If $f: U \rightarrow \mathbb{C}$ is holomorphic, then $f \circ \varphi^{-1}$ is holomorphic on the disk and

$$
f\left(\varphi^{-1}(w)\right)=\sum_{n=0}^{\infty} a_{n} w^{n}, \quad|w|<1,
$$

where $a_{n}$ is the $n$-th Taylor coefficient of $f \circ \varphi^{-1}$ at the origin. In particular, $f \circ \varphi^{-1}$ has a contact of order $m$ with the polynomial defined by $P(w)=\sum_{n=0}^{m} a_{n} w^{n}$ at the origin. As $\varphi$ is a conformal equivalence, we have that $f=f \circ \varphi^{-1} \circ \varphi$ has a contact of order $m$ with the function

$$
P \circ \varphi=\sum_{n=0}^{m} a_{n} \varphi^{n} \quad \text { at } z=\varphi^{-1}(0) .
$$

In particular, the first $m$ derivatives of $f$ and $\sum_{n=0}^{m} a_{n} \varphi^{n}$ agree at $z$. The lemma follows by applying this construction to the function $f(w)=(w-z)^{k}$.

The following is a slight generalization of [Rochberg 1996, Proposition 3.1]:

Proposition 2. Suppose $1 \leq n, k<m$. Then,

(a) The map $\imath_{n, m}: \mathscr{L}^{(n)} \rightarrow \mathscr{L}^{(m)}$ is bounded.

(b) The map $\pi_{m, k}: \mathscr{L}^{(m)} \rightarrow \mathscr{L}^{(k)}$ is an "isometric" quotient.

Proof. Part (b) is obvious. To prove (a) we must prove that there is a constant $M$ such that if $\left(x_{n}, \ldots, x_{1}\right)$ is the list of Taylor coefficients of some $f \in \mathscr{F}$, then there is another $g \in \mathscr{F}$ whose coefficients are $\left(x_{n}, \ldots, x_{1}, 0, \ldots, 0\right)$ with $\|g\|_{\mathscr{F}} \leq M\|f\|_{\mathscr{F}}$. Set $k=m-n$ and apply Lemma 1 to get a polynomial $\Pi=\sum_{i=0}^{m-1} a_{i} \varphi^{i}$ such that $\widehat{\Pi}[i ; z]=\delta_{i k}$ for $0 \leq i<n+k$ and take $M=\sum_{i=0}^{n+k-1}\left|a_{i}\right|$. Now, if $f \in \mathscr{F}$ and $g=\Pi f$, then $\|g\|_{\mathscr{F}} \leq M\|f\|_{\mathscr{F}}$. Moreover, for $i \in[0, n+k)$,

$$
\hat{g}[i ; z]=(\widehat{\Pi f})[i ; z]=\sum_{j=0}^{i} \widehat{\Pi}[j ; z] \cdot \hat{f}[i-j ; z],
$$

by the Leibniz rule. Hence, $\hat{g}[i ; z]=0$ if $i<k$ and $\hat{g}[i ; z]=\hat{f}[i-k ; z]$ if $i \geq k$, as required.

3.3. Exactness. From now on, we will omit the names $\imath_{m, n}$ and $\pi_{m, k}$, so unlabeled arrows $\mathscr{X}^{(n)} \rightarrow \mathscr{L}^{(m)}$ must be understood to be $\iota_{n, m}$ if $n \leq m$ and $\pi_{n, m}$ if $n \geq m$, unless otherwise declared. With these conventions, the aim of this section is to prove that, given integers $n$ and $k$, the "obvious" sequence $0 \longrightarrow \mathscr{X}^{(n)} \longrightarrow \mathscr{X}^{(n+k)} \longrightarrow \mathscr{X}^{(k)} \longrightarrow 0$ is exact.

First of all, observe that the various possible sequences passing through a given $\mathscr{L}^{(m)}$ are compatible in the sense that if $m=k+n=i+j$, with $k<i$ say, then the 
following diagram is commutative:

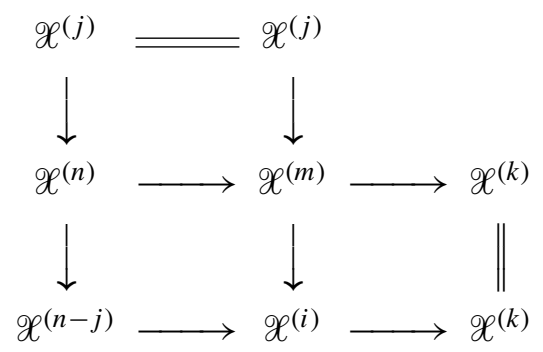

The key point is isolated in the next lemma.

Lemma 3. If $(x, 0, \ldots, 0) \in \mathscr{X}^{(k+1)}$, then $x \in \mathscr{X}^{(1)}$.

Proof. Pick $x \in W$ and suppose $(x, 0, \ldots, 0) \in \mathscr{X}^{(k+1)}$. Let us take $f \in \mathscr{F}$ such that $\hat{f}[i ; z]=0$ for $i<k$ and $x=\hat{f}[k ; z]$. Then $f$ has a zero of order $k-1$ at $z$ and it can be written as $f=\varphi^{k} g$, where $g: U \rightarrow W$ is analytic. It follows from (b) that $g \in \mathscr{F}$ and $\|g\|_{\mathscr{F}}=\|f\|_{\mathscr{F}}$. But,

$x=\hat{f}[k ; z]=\left(\widehat{\varphi^{k} g}\right)[k ; z]=\sum_{i=0}^{k}\left(\widehat{\varphi^{k}}\right)[i ; z] \cdot \hat{g}[k-i ; z]=\frac{\left(\varphi^{k}\right)^{(k)}(z) g(z)}{k !}=\varphi^{\prime}(z)^{k} g(z)$ and so $x \in \mathscr{L}^{(1)}$.

Theorem 4. The sequence $0 \longrightarrow \mathscr{X}^{(n)} \longrightarrow \mathscr{X}^{(n+k)} \longrightarrow \mathscr{X}^{(k)} \longrightarrow 0$ is exact.

Proof. The proof proceeds by induction on $m=n+k$. The previous lemma shows that for every $m \in \mathbb{N}$, the sequence $0 \longrightarrow \mathscr{X}^{(1)} \longrightarrow Z^{(m)} \longrightarrow \mathscr{X}^{(m-1)} \longrightarrow 0$ is exact. By the induction hypothesis, the sequence $0 \longrightarrow \mathscr{X}^{(n-1)} \longrightarrow \mathscr{X}^{(m-1)} \longrightarrow \mathscr{X}^{(k)} \longrightarrow 0$ is also exact. The compatibility of such sequences yields the commutative diagram:

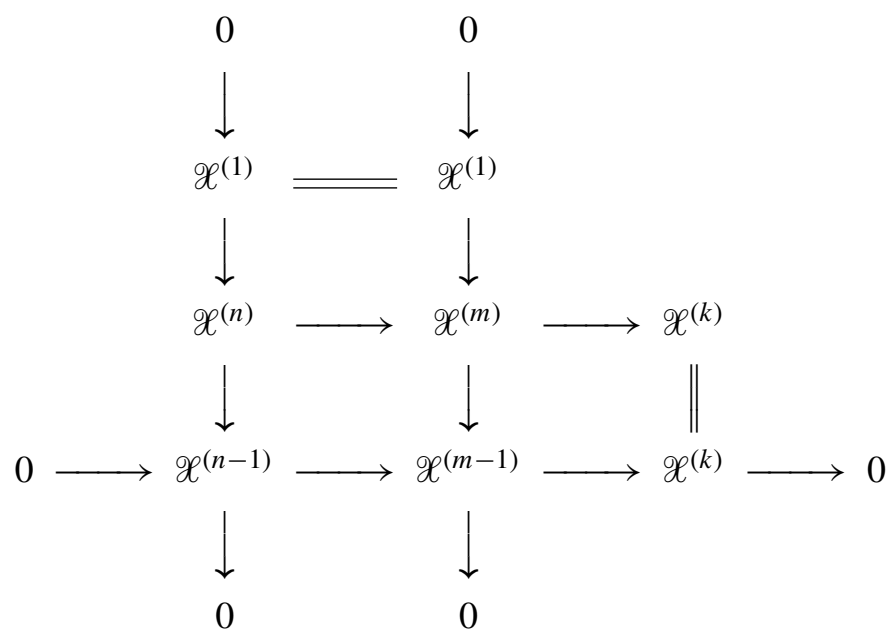

and a simple arrow chase shows that the middle sequence must also be exact. 
Corollary 5. If $\left(x_{n}, \ldots, x_{1}, 0, \ldots, 0\right) \in \mathscr{L}^{(n+k)}$, then $\left(x_{n}, \ldots, x_{1}\right) \in \mathscr{L}^{(n)}$.

This implies that $\left\|\left(x_{n}, \ldots, x_{1}, 0, \ldots, 0\right)\right\|_{\mathscr{Q}(n+k)}$ is equivalent to $\left\|\left(x_{n}, \ldots, x_{1}\right)\right\|_{\mathscr{L}(n)}$, although we will not pursue any bound here.

A new look can be had now at (5) to exploit its form to study the splitting of the exact sequences it contains. After Theorem 4 the diagram has become:

(6)

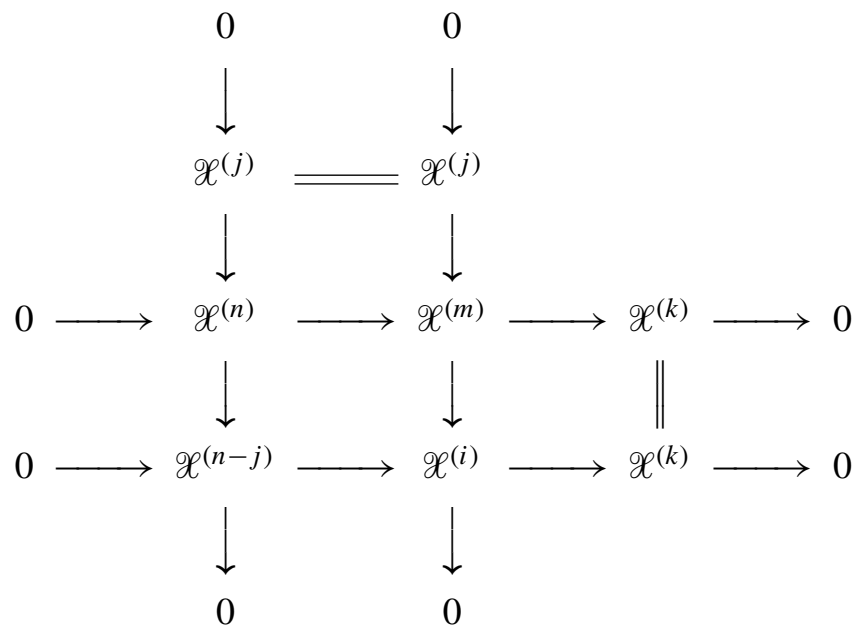

Thus, if the middle horizontal sequence splits, then so does the lower one, and if the middle vertical sequence splits, then so does the vertical sequence on the left. Putting together these two pieces, one gets the commutative diagram:

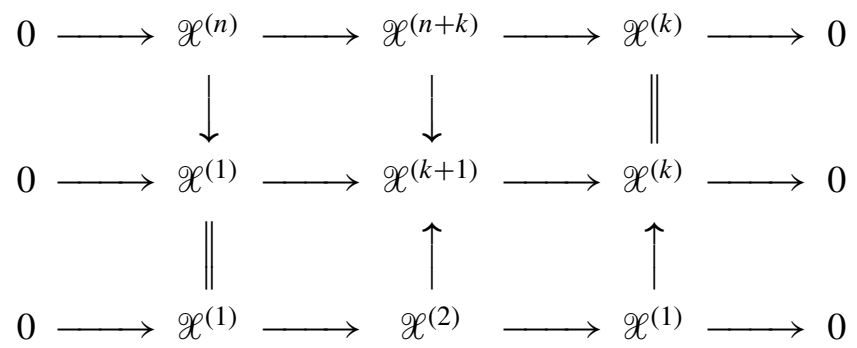

which immediately yields the following corollary.

Corollary 6. If the sequence $0 \longrightarrow \mathscr{X}^{(n)} \longrightarrow \mathscr{X}^{(n+k)} \longrightarrow \mathscr{X}^{(k)} \longrightarrow 0$ is nontrivial for $n=k=1$, then it is nontrivial for all integers $n$ and $k$.

3.4. An isometric variant. There is another form for the short exact sequences $0 \rightarrow \mathscr{X}^{(n)} \rightarrow \mathscr{X}^{(n+k)} \rightarrow \mathscr{X}^{(k)} \rightarrow 0$ which is even easier to describe in abstract terms. Consider again the quotient spaces

$$
Q_{z}^{(n)}=\mathscr{F} / \bigcap_{i<n} \operatorname{ker} \delta_{z}^{i} \quad \text { for } n \in \mathbb{N} .
$$


These spaces are isometric to the corresponding $\mathscr{X}_{z}^{(n)}$ via Taylor coefficients, but we do not need this fact at this moment. Fix integers $n$ and $k$. It is clear that there is a natural quotient map from $Q_{z}^{(n+k)}$ onto $Q_{z}^{(k)}$ that we shall not even label. Less obvious is that the kernel of this map is isometric to $\mathscr{X}_{z}^{(n)}$, although this time the isometry is not "natural". To see this, let us fix a conformal equivalence $\varphi: U \rightarrow \mathbb{D}$ having a (single) zero at $z$. (We observe that if $\Pi$ is another conformal equivalence with $\Pi(z)=0$, then $\Pi=\lambda \varphi$, where $\lambda \in \mathbb{T}$; thus, $\varphi$ is unique if we insist that $\varphi^{\prime}(0)$ is real and positive.)

Now, recall that $f \in \bigcap_{i<k} \operatorname{ker} \delta_{z}^{i}$ if and only if there is a (necessarily unique) $g \in \mathscr{F}_{F}$ such that $f=\varphi^{k} g$ and one has $\|f\|_{\mathscr{F}}=\|g\|_{\mathscr{F}}$, by (b). Therefore, it is clear that the map $f \in \mathscr{F} \mapsto \varphi^{k} f \in \mathscr{F}$ induces an isometry of $Q_{z}^{(n)}$ into $Q_{z}^{(n+k)}$ whose range is

$$
\operatorname{ker}\left(Q_{z}^{(n+k)} \rightarrow Q_{z}^{(k)}\right)
$$

As a consequence, the space $Q_{z}^{(n+k)}$ is an "isometric" twisted sum of $Q_{z}^{(n)}$ and $Q_{z}^{(k)}$. More precisely, the short sequence

$$
0 \longrightarrow Q_{z}^{(n)} \stackrel{\varphi^{k}}{\longrightarrow} Q_{z}^{(n+k)} \longrightarrow Q_{z}^{(k)} \longrightarrow 0
$$

is exact.

From now on, we will omit the base point $z$, which is understood. As before, the decompositions of a given $Q_{z}^{(m)}$ into a twisted sum of the preceding spaces $Q_{z}^{(n)}$ are all compatible in the sense that if $m=k+n=i+j$, with $k<i$, then the following diagram is commutative:

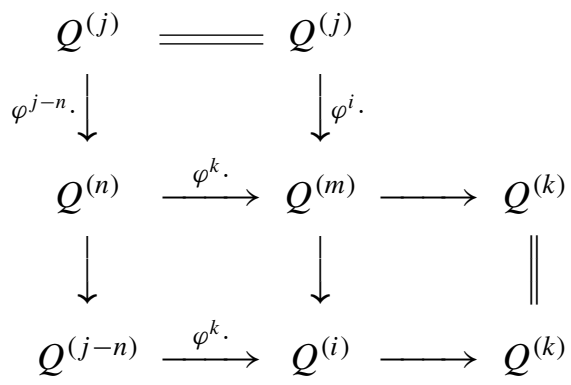

It is interesting to compare the sequence (7) to that appearing in Theorem 4. To this end, we observe that, after identifying $\mathscr{C}^{(m)}$ and $Q^{(m)}$ through Taylor coefficients, the operator $\mathscr{X}^{(n)} \rightarrow \mathscr{X}^{(n+k)}$ which corresponds to $\imath_{n, n+k}: \mathscr{X}^{(n)} \rightarrow \mathscr{X}^{(n+k)}$ is just multiplication by $\Pi$, where $\Pi$ is the polynomial appearing in the proof of Proposition 2(a), that is,

$$
\Pi=\sum_{0 \leq i<n+k} a_{i} \varphi^{i}, \quad \text { with } \widehat{\Pi}[i ; z]=\delta_{i k} \text { for } 0 \leq i<n+k .
$$


Clearly, $a_{i}=0$ for $0 \leq i<k$, so $\Pi=\varphi^{k} \psi$, where $\psi=\sum_{k \leq i<n+k} a_{i} \varphi^{i-k}$. Thus, the following diagram is commutative:

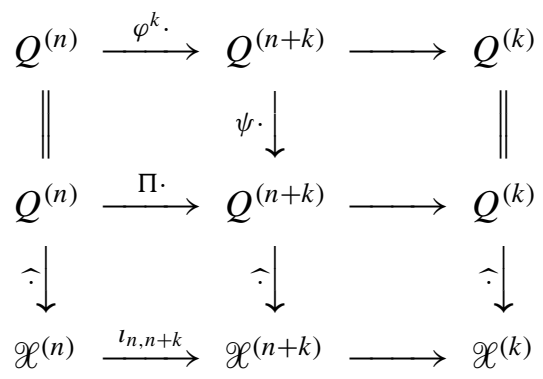

It follows from the 3-lemma (see, for instance, [Hilton and Stammbach 1971, Lemma 1.1]) and the open mapping theorem that multiplication by $\psi$ induces an automorphism of $Q^{(n+k)}$. So, in the preceding diagram, the first row is equivalent to the second one, and both are "isomorphically equivalent" (in the language of [Castillo and Moreno 2004, p. 256]) to the third one, which means that the three sequences have the same "isomorphic" properties.

3.5. The space $\mathscr{L}^{(n+k)}$ as a twisted sum of $\mathscr{L}^{(n)}$ and $\mathscr{L}^{(k)}$. It is a part of the (by now) classical theory of twisted sums as developed by Kalton [1978, Proposition 3.3; Kalton and Peck 1979, Theorem 2.4] that if $A$ and $C$ are Banach or quasi-Banach spaces, then every short exact sequence $0 \rightarrow A \rightarrow B \rightarrow C \rightarrow 0$ arises, up to equivalence, from a quasilinear map from $C$ to $A$. Thus, in view of Theorem 4, given integers $k$ and $n$, there must be some quasilinear map $\Omega_{k, n}$ associated to the exact sequence $0 \rightarrow \mathscr{X}^{(n)} \rightarrow \mathscr{X}^{(n+k)} \rightarrow \mathscr{X}^{(k)} \rightarrow 0$. From an abstract point of view, the description of $\Omega_{k, n}$ is rather easy: fix some (small) $\varepsilon>0$. Now, given $x=$ $\left(x_{k-1}, \ldots, x_{0}\right) \in \mathscr{L}^{(k)}$, select (homogeneously) $f \in \mathscr{F}$ such that $\|f\| \leq(1+\varepsilon)\|x\|_{\mathscr{L}_{(n)}}$ and $\hat{f}[i ; z]=x_{i}$ for $0 \leq i<k$, and define $\Omega_{k, n}: \mathscr{L}^{(k)} \rightarrow W^{n}$ by letting

$$
\Omega_{k, n}(x)=(\hat{f}[n+k-1 ; z], \ldots, \hat{f}[k ; z]) .
$$

Following the uses of the theory, the twisted sum space (sometimes known as the derived space) is then defined by

$$
\mathscr{L}^{(n)} \oplus_{\Omega_{k, n}} \mathscr{L}^{(k)}=\left\{(y, x) \in W^{n} \times W^{k}: x \in \mathscr{X}^{(k)}, y-\Omega_{k, n}(x) \in \mathscr{L}^{(n)}\right\},
$$

endowed with the quasinorm

$$
\|(y, x)\|_{\Omega_{k, n}}=\left\|y-\Omega_{k, x}(x)\right\|_{\mathscr{P}(n)}+\|x\|_{\mathscr{\mathscr { C }}(k)} .
$$

Of course, it has not yet been proved either that $\Omega_{k, n}$ is quasilinear or that (9) defines a quasinorm. We may skip these steps since we have the following.

Proposition 7. The spaces $\mathscr{L}^{(n)} \oplus_{\Omega_{k, n}} \mathscr{L}^{(k)}$ and $\mathscr{L}^{(n+k)}$ agree. Their quasinorms are equivalent. 
Proof. Suppose $(y, x)=\left(y_{n-1}, \ldots, y_{0}, x_{k-1}, \ldots, x_{0}\right) \in \mathscr{X}^{(n+k)}$ so that there is $F \in \mathscr{F}_{F}$ whose list of Taylor coefficients begins with $(y, x)$. Then $x \in \mathscr{X}^{(k)}$ and $\left(\Omega_{k, n}(x), x\right) \in \mathscr{L}^{(n+k)}$, so $(y, x)-\left(\Omega_{k, n}(x), x\right)=\left(y-x, \Omega_{k, n}(x), 0\right)$ belongs to $\mathscr{X}^{(n+k)}$ and by Lemma 3 we have $y-\Omega_{k, n}(x) \in \mathscr{X}^{(n)}$. Regarding the involved norms, $\left\|y-\Omega_{k, x}(x)\right\|_{\mathscr{L}(n)} \leq C\left(\|(y, x)\|_{\mathscr{P}^{(n+k)}}-\left\|\left(\Omega_{k, n}(x), x\right)\right\|_{\mathscr{L}^{(n+k)}}\right) \leq(C+1)\|(y, x)\|_{\mathscr{L}^{(n+k)}}$, where $C$ is the constant implicit in Lemma 3. Hence,

$$
\|(y, x)\|_{\Omega_{k, n}} \leq(C+2)\|(y, x)\|_{\mathscr{P}^{(n+k)}} .
$$

As for the other containment, suppose $(y, x) \in \mathscr{X}^{(n)} \oplus_{\Omega_{k, n}} \mathscr{X}^{(k)}$, that is, $x \in \mathscr{X}^{(k)}$ and $y-\Omega_{k, n}(x) \in \mathscr{L}^{(n)}$. Then if $f$ is the function associated to $\Omega_{k, n}(x)$ as in (8) and $g \in \mathscr{F}$ is almost optimal for $y-\Omega_{k, n}(x) \in \mathscr{L}^{(n)}$, taking $\Pi$ as in Lemma 1 , we have that $(y, x)$ is the list of Taylor coefficients of $F=f+\Pi \cdot g$, so $(y, x) \in \mathscr{X}^{(n+k)}$ and

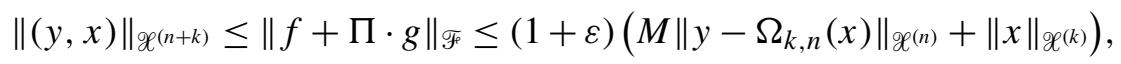

where $M$ is as in the proof of Proposition 2(a).

\section{Singularity of the exact sequences of derived spaces}

Recall that an operator is said to be strictly singular if its restriction to an infinite dimensional subspace of its domain is never an isomorphism; and that an operator $u: A \rightarrow B$ is strictly cosingular if for every infinite codimensional subspace $C$ of $B$ the composition $\pi \circ u: A \rightarrow B \rightarrow B / C$ fails to be onto; equivalently, $u^{*}: B^{*} \rightarrow A^{*}$ is not an isomorphism when restricted to any weakly- closed infinite-dimensional subspace of $B^{*}$. Strictly singular operators were introduced by Kato [1958] and strictly cosingular operators by Pełczyński [1965].

An exact sequence is said to be singular when the quotient map is strictly singular and will be called cosingular when the embedding is strictly cosingular. We refer the reader to [Castillo and Moreno Salguero 2007; Cabello Sánchez et al. 2012] for some steps into the theory of singular and cosingular sequences. The Kalton-Peck sequences $0 \rightarrow \ell_{p} \rightarrow Z_{p} \rightarrow \ell_{p} \rightarrow 0$ are singular for all $p \in(0, \infty)$ and cosingular at least for $p \in(1, \infty)$. We need the following result.

Lemma 8. Assume one has a commutative diagram

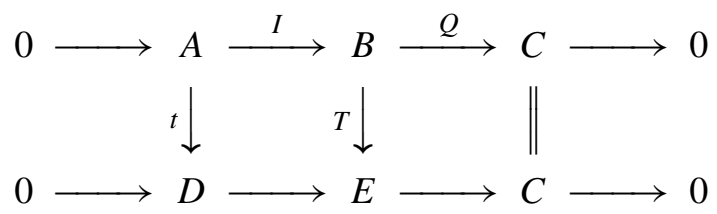

with exact rows. If both $Q$ and $t$ are strictly singular, then $T$ is strictly singular. 
Proof. We need the following characterization of strictly singular quotient maps. Let $B$ be a Banach space and $A$ a closed subspace of $B$. Then the quotient map $Q: B \rightarrow B / A$ is strictly singular if and only if for every infinite-dimensional subspace $B^{\prime} \subset B$ there is an infinite-dimensional $A^{\prime} \subset A$ and a compact (actually nuclear) operator $K: A^{\prime} \rightarrow B$ such that $I+K$ embeds $A^{\prime}$ into $B^{\prime}$ isomorphically. This maybe folklore; see [Castillo et al. 2012, Proposition 3.2] for an explicit proof. A certainly classical result establishes that an operator $t: A \rightarrow D$ is strictly singular if given any infinite dimensional subspace $A^{\prime} \subset A$ and $\varepsilon>0$ there is a further infinite dimensional subspace $A^{\prime \prime} \subset A^{\prime}$ such that $\left\|\left.t\right|_{A^{\prime \prime}}\right\|<\varepsilon$. Both things together yield that given $B^{\prime} \subset B$ there is $A^{\prime \prime} \subset A^{\prime} \subset A$ such that $I+K: A^{\prime \prime} \rightarrow B^{\prime}$ is an into isomorphism and $\left\|\left.t\right|_{A^{\prime \prime}}\right\|<\varepsilon$. There is no loss of generality assuming that $\left\|\left.K\right|_{A^{\prime \prime}}\right\|<\varepsilon$. Therefore, $\left\|\left.T\right|_{(I+K)\left(A^{\prime \prime}\right)}\right\|=\left\|\left.t\right|_{A^{\prime \prime}}+\left.T K\right|_{A^{\prime \prime}}\right\|<(1+\|T\|) \varepsilon$.

We thus obtain the "strictly singular counterpart" to Corollary 6:

Proposition 9. If the natural quotient map $\mathscr{X}^{(2)} \rightarrow \mathscr{L}^{(1)}$ is strictly singular, then so is $\mathscr{L}^{(n)} \rightarrow \mathscr{L}^{(k)}$ for every $n>k$.

Proof. Note that if $n>m>k$, then $\mathscr{L}^{(n)} \rightarrow \mathscr{L}^{(k)}$ is $\mathscr{L}^{(n)} \rightarrow \mathscr{L}^{(m)}$ followed by $\mathscr{X}^{(m)} \rightarrow \mathscr{X}^{(k)}$. As the composition of a strictly singular operator with any operator is again strictly singular, we have that the proposition is trivial if $k=1$ and also that one can assume $n=k+1$. We shall prove that $\mathscr{L}^{(k+1)} \rightarrow \mathscr{L}^{(k)}$ is strictly singular by induction on $k \in \mathbb{N}$. There is nothing to prove for $k=1$, so assume $k>1$. Since one has the commutative diagram

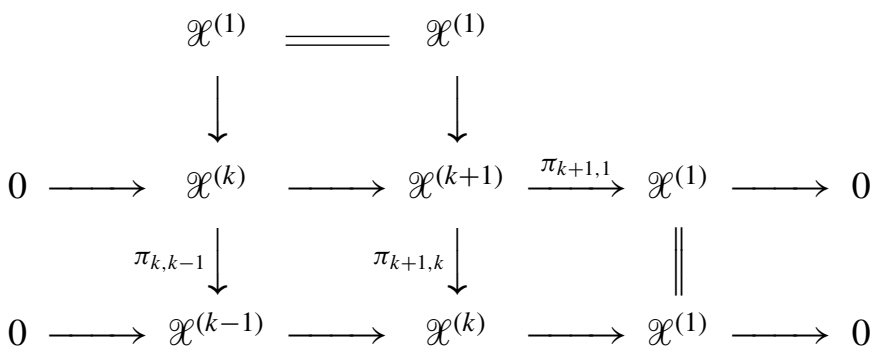

But $\pi_{k+1,1}$ is strictly singular and so is $\pi_{k, k-1}$, by the induction hypothesis. Thus, the result follows from Lemma 8 .

We omit the proofs of the dual results:

Lemma 10. Assume one has a commutative diagram

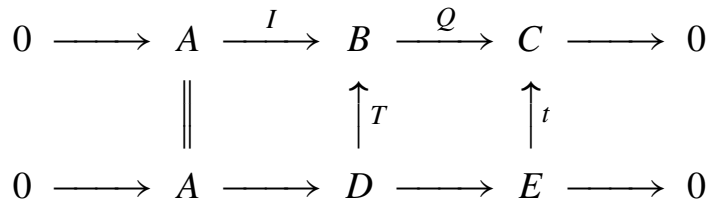

with exact rows. If both $I$ and $t$ are strictly cosingular, then $T$ is strictly cosingular. 
Corollary 11. If the inclusion map $\mathscr{X}^{(1)} \rightarrow \mathscr{X}^{(2)}$ is strictly cosingular, then so is $\mathscr{L}^{(k)} \rightarrow \mathscr{L}^{(n)}$ for every $k<n$.

\section{Applications to Hilbert spaces}

5.1. The quasilinear map associated to twisted Kalton-Peck spaces. Some results in this section are, essentially, in [Rochberg 1996, Section 6B]. Let us consider the following variation of the Calderón space associated to the Banach couple $\left(\ell_{\infty}, \ell_{1}\right)$ which is designed to simplify the computation of extremals. Take $U=\mathbb{S}=\{z \in \mathbb{C}$ : $0<\Re z<1\}$, with $W=\ell_{\infty}$, and let $\mathscr{F}$ be the space of analytic functions $F: \mathbb{S} \rightarrow \ell_{\infty}$ having the following properties:

(1) $F$ extends to a $\sigma\left(\ell_{\infty}, \ell_{1}\right)$ continuous function on $\bar{S}$ that we denote again by $F$.

(2) $\|F\|_{\mathscr{F}}=\sup \left\{\|F(i t)\|_{\infty},\|F(1+i t)\|_{1}: t \in \mathbb{R}\right\}<\infty$.

Let $\left(\mathscr{L}_{z}\right)_{z \in \mathbb{S}}$ denote the analytic family induced by $\mathscr{F}_{\text {. }}$. Then, of course, $\mathscr{L}_{z}=$ $\left[\ell_{\infty}, \ell_{1}\right]_{\theta}=\ell_{p}$, where $\theta=\Re z$ and $p=\frac{1}{\theta}$ for $\theta \in(0,1)$, and, in particular, $\mathscr{Z}_{z}=\ell_{2}$ for $z=\frac{1}{2}$. For the remainder of this section, we fix $z=\frac{1}{2}$ as the base point.

If $x$ is normalized in $\ell_{2}$, then $F_{x}(z)=u|x|^{2 z}$ is normalized in $\mathscr{F}_{F}$ (although it does not belong to $\mathscr{C}\left(\ell_{\infty}, \ell_{1}\right)$ in general), and one has $F_{x}\left(\frac{1}{2}\right)=x$, where $x=u|x|$ is the "polar decomposition" of $x$. Now,

$$
F_{x}=u|x||x|^{2 z-1}=x|x|^{2(z-1 / 2)}=x \sum_{n=0}^{\infty} \frac{2^{n} \log ^{n}|x|}{n !}\left(z-\frac{1}{2}\right)^{n},
$$

and

$$
\hat{F}_{x}\left[n ; \frac{1}{2}\right]=\frac{2^{n} x \log ^{n}|x|}{n !},
$$

if $\|x\|_{2}=1$. For arbitrary $x \in \ell_{2}$, we have, by homogeneity,

$$
\hat{F}_{x}\left[n ; \frac{1}{2}\right]=\frac{2^{n} x}{n !} \log ^{n} \frac{|x|}{\|x\|_{2}} .
$$

In particular,

$$
\begin{aligned}
\Omega_{1, n}(x) & =\left(\hat{F}_{x}\left[n ; \frac{1}{2}\right], \ldots, \hat{F}_{x}\left[1 ; \frac{1}{2}\right]\right) \\
& =x\left(\frac{2^{n}}{n !} \log ^{n} \frac{|x|}{\|x\|_{2}}, \ldots, 2 \log ^{2} \frac{|x|}{\|x\|_{2}}, 2 \log \frac{|x|}{\|x\|_{2}}\right),
\end{aligned}
$$

which allows us to describe the corresponding spaces $\mathscr{L}^{(n)}$, for small $n$, as follows. First, we have

$$
\mathscr{L}^{(2)} \approx \ell_{2} \oplus_{\Omega_{1,1}} \ell_{2}=\left\{(y, x):\left\|y-2 x \log \frac{|x|}{\|x\|_{2}}\right\|_{2}+\|x\|_{2}<\infty\right\},
$$


which is isomorphic to the Kalton-Peck space $Z_{2}$ [1979, Section 6], which was defined there by the quasilinear map $x \mapsto x \log \left(\|x\|_{2} /|x|\right)=-\frac{1}{2} \Omega_{1,1}(x)$. Also,

$$
\mathscr{L}^{(3)} \approx \mathscr{L}^{(2)} \oplus_{\Omega_{1,2}} \ell_{2} \approx\left(\ell_{2} \oplus_{\Omega_{1,1}} \ell_{2}\right) \oplus_{\Omega_{1,2}} \ell_{2},
$$

and the norm of $\mathscr{L}^{(3)}$ is equivalent to

$$
\|(z, y, x)\|_{\Omega_{1,2}}=\left\|\left(z-2 x \log ^{2} \frac{|x|}{\|x\|_{2}}, y-2 x \log \frac{|x|}{\|x\|_{2}}\right)\right\|_{\Omega_{1,1}}+\|x\|_{2} .
$$

We will also finally display the quasilinear map $\Omega_{2,2}$ that allows one to represent $\mathscr{L}^{(4)}$ as a twisted sum of $\mathscr{L}^{(2)}$ with itself. After all, this was the starting point of this research. Let $\varphi: \mathbb{S} \rightarrow \mathbb{D}$ be conformal equivalence vanishing at $z_{0}=\frac{1}{2}$ and let $\Pi=\sum_{1 \leq i \leq 3} a_{i} \varphi^{i}$ be such that $\widehat{\Pi}\left[i ; \frac{1}{2}\right]=\delta_{1 i}$ for $0 \leq i \leq 3$. Given $(y, x) \in \mathscr{L}^{(2)}$, we construct an allowable $F_{(y, x)} \in \mathscr{F}$ as follows. Let $F_{x}$ and $F_{(y-\Omega(x))}$ be extremals for $x$ and $y-\Omega(x)$, respectively, where $\Omega(x)=\Omega_{1,1}(x)=F_{x}^{\prime}\left(\frac{1}{2}\right)=2 x \log \left(|x| /\|x\|_{2}\right)$. Put

$$
F_{(y, x)}=\Pi \cdot F_{(y-\Omega(x))}+F_{x} .
$$

Then, $F_{(y, x)}\left(\frac{1}{2}\right)=x, F_{(y, x)}^{\prime}\left(\frac{1}{2}\right)=y$, and

$$
\left\|F_{(y, x)}\right\|_{\mathscr{F}} \leq\left\|\Pi \cdot F_{(y-\Omega(x))}\right\|_{\mathscr{F}}+\left\|F_{x}\right\|_{\mathscr{F}} \leq\|\Pi\|_{\infty}\left(\|y-\Omega(x)\|_{2}+\|x\|_{2}\right),
$$

where $\|\Pi\|_{\infty} \leq\left|a_{1}\right|+\left|a_{2}\right|+\left|a_{3}\right|$, and we may define

$$
\Omega_{2,2}(y, x)=\left(\hat{F}_{(y, x)}\left[3 ; \frac{1}{2}\right], \hat{F}_{(y, x)}\left[2 ; \frac{1}{2}\right]\right) .
$$

By the construction of $\Pi$, we have

and thus

$$
\begin{aligned}
& \hat{F}_{(y, x)}\left[2 ; \frac{1}{2}\right]=\hat{F}_{(y-\Omega(x))}\left[1 ; \frac{1}{2}\right]+\hat{F}_{x}\left[2 ; \frac{1}{2}\right], \\
& \hat{F}_{(y, x)}\left[3 ; \frac{1}{2}\right]=\hat{F}_{(y-\Omega(x))}\left[2 ; \frac{1}{2}\right]+\hat{F}_{x}\left[3 ; \frac{1}{2}\right],
\end{aligned}
$$

$$
\begin{aligned}
\Omega_{2,2}(y, x)=2\left((y-\Omega x) \log ^{2} \frac{|y-\Omega x|}{\|y-\Omega x\|_{2}}\right. & +\frac{2 x}{3} \log ^{3} \frac{|x|}{\|x\|_{2}}, \\
& \left.(y-\Omega x) \log \frac{|y-\Omega x|}{\|y-\Omega x\|_{2}}+x \log ^{2} \frac{|x|}{\|x\|_{2}}\right) .
\end{aligned}
$$

At this point we cannot help to mention a few very accurate comments of the referee: "Speaking of computational details [...], we have that $\mathscr{L}^{(4)}$ is equivalent to both a twisted sum of $\mathscr{X}^{(2)}$ with $\mathscr{L}^{(2)}$ and to a twisted sum of $\mathscr{L}^{(3)}$ with $\mathscr{X}^{(1)}$. Presumably that can be seen in the case of $\ell_{2}$ by working directly with the explicit formulas in Section 5.1 and doing some estimation. In fact, given that the twisted direct sum of $\mathscr{L}^{(2)}$ with $\mathscr{L}^{(1)}$ is not automatically equivalent to the twisted sum of $\mathscr{L}^{(1)}$ with $\mathscr{L}^{(2)}$, the fact that these are equivalent would also be visible through the formulas in [that section]. If the authors have done the computations or if they 
choose to do them, and if the result seems to carry any intuitive insight, they would make a good inclusion." To this plot the referee foresees: "My first thought is that the answer is 'Yes, the computations can be done. Yes, the result is a mess. No, it doesn't seem to give any insights'."

Indeed, the computations were done a long time ago and are a mess, generally speaking. However, the "3D-case" is crystalline since in $\mathscr{L}^{(3)}=\ell_{2} \oplus_{\Omega_{2,1}} \mathscr{L}^{(2)}=$ $\mathscr{L}^{(2)} \oplus_{\Omega_{1,2}} \ell_{2}$ the quasinorms induced by $\Omega_{2,1}$ and $\Omega_{1,2}$ actually agree. To check this first observe that, letting $\Lambda(x)=\log \left(|x| /\|x\|_{2}\right)$, one can rewrite (11) as

$$
\|(z, y, x)\|_{\Omega_{1,2}}=\|z-\Omega(x) \Lambda(x)-\Omega(y-\Omega(x))\|_{2}+\|y-\Omega(x)\|_{2}+\|x\|_{2}
$$

To compute $\|(z, y, x)\|_{\Omega_{2,1}}$ let us take a look at $\Omega_{2,2}$ and notice that $\Omega_{2,1}$ just "forgets" the first coordinate. Hence,

$$
\Omega_{2,1}(y, x)=\Omega(y-\Omega(x))+\Omega(x) \Lambda(x)
$$

and

$$
\begin{aligned}
\|(z, y, x)\|_{\Omega_{2,1}} & =\left\|z-\Omega_{2,1}(y, x)\right\|_{2}+\|(y, x)\|_{\Omega} \\
& =\|z-\Omega(y-\Omega(x))-\Omega(x) \Lambda(x)\|_{2}+\|y-\Omega(x)\|_{2}+\|x\|_{2} \\
& =\|(z, y, x)\|_{\Omega_{1,2}} .
\end{aligned}
$$

5.2. The 3-space problem for twisted Hilbert spaces. We are now ready for the first concrete application. Recall that a twisted Hilbert space is a twisted sum of Hilbert spaces.

Proposition 12. The space $\mathscr{E}^{(n)}$ is a twisted Hilbert space if and only if $n=1,2$.

Proof. The $n$-th type 2 constant $a_{n, 2}(X)$ of a (quasi-)Banach space $X$ is defined as the infimum of those $C$ such that for every $x_{1}, \ldots x_{n} \in X$ one has

$$
\left(\int_{0}^{1}\left\|\sum_{i=1}^{n} r_{i}(t) x_{i}\right\|^{2} d t\right)^{\frac{1}{2}} \leq C\left(\sum_{i=1}^{n}\left\|x_{i}\right\|^{2}\right)^{\frac{1}{2}},
$$

where $\left(r_{n}\right)$ is the sequence of Rademacher functions.

To prove that $\mathscr{L}^{(3)}$ does not embed in any twisted Hilbert space, we will work with the equivalent quasinorm given by (11). Let $\left(e_{i}\right)$ be the unit basis of $\ell_{2}$ and take $x_{i}=\left(0,0, e_{i}\right)$. These are normalized vectors, which makes $\left(\sum_{i=1}^{n}\left\|x_{i}\right\|^{2}\right)^{1 / 2}=\sqrt{n}$. On the other hand,

$$
\left\|\sum_{i=1}^{n} \pm x_{i}\right\|_{\Omega_{1,2}}=\sqrt{n}\left(1+\log ^{2} n\right) .
$$

Hence, the type 2 constants of $\mathscr{L}^{(3)}$ cannot satisfy $a_{n, 2} \leq K \log n$, and this estimate holds in any twisted Hilbert space by [Kalton and Peck 1979, Theorem 6.2(a)]. 
Corollary 13. "To be a twisted Hilbert space" is not a 3-space property.

This corollary answers a question posed to us by David Yost a long time ago [Castillo and González 1997, p. 95]; the first author showed [Cabello Sánchez 1999] that "to be a subspace of a twisted Hilbert space" is not a 3-space property. Since $\mathscr{L}^{(n)}$ is isomorphic to its dual [Rochberg 1996, Section 4] and the dual of any twisted Hilbert space is again a twisted Hilbert space, we see that $\mathscr{L}^{(n)}$ is a quotient of a twisted Hilbert space if and only if $n=1,2$.

Thus, in the situation described in Section 5.1, recall that for $\mathscr{F}=\mathscr{F}\left(\ell_{\infty}, \ell_{1}\right)$ one gets $\mathscr{L}^{(1)}=\ell_{2}$ and $\mathscr{L}^{(2)}$ is isomorphic to the Kalton-Peck space $Z_{2}$, and, actually, the extension $0 \rightarrow \ell_{2} \rightarrow \mathscr{L}^{(2)} \rightarrow \ell_{2} \rightarrow 0$ is isomorphically - and even "projectively", compare [Kalton and Peck 1979, Definition 2.1(b)] — equivalent to the Kalton-Peck sequence $0 \rightarrow \ell_{2} \rightarrow Z_{2} \rightarrow \ell_{2} \rightarrow 0$, which has strictly singular quotient map and strictly cosingular inclusion (see Theorem 6.4 of the same reference). Thus applying the results of Section 4 we obtain the following.

Proposition 14. The exact sequences $0 \rightarrow \mathscr{L}^{(k)} \rightarrow \mathscr{L}^{(n+k)} \rightarrow \mathscr{L}^{(n)} \rightarrow 0$ are singular and cosingular for all integers $n, k$.

As a direct application we get:

Proposition 15. If $k>1$, then $\mathscr{L}^{(k)}$ does not contain complemented copies of $\ell_{2}$. Proof. By [Kalton and Peck 1979, Corollary 6.7], $\mathscr{L}^{(2)}=Z_{2}$ has no complemented subspaces isomorphic to $\ell_{2}$. Now, if one has an exact sequence

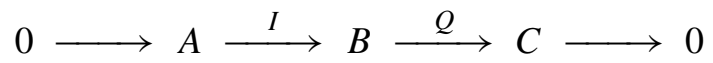

with $Q$ strictly singular and $A$ not containing $\ell_{2}$ complemented, then $B$ does not contain $\ell_{2}$ complemented: assume otherwise that $B$ has a subspace $B^{\prime}$ which is isomorphic to $\ell_{2}$ and is complemented in $B$ through a projection $P$. (Without loss of generality we may assume that $A=\operatorname{ker} Q$ and that $I$ is the inclusion map.) Since $Q$ is strictly singular, there exists an infinite dimensional subspace $A^{\prime} \subset A$ and a nuclear operator $K: A^{\prime} \rightarrow B$ such that $I-K: A^{\prime} \rightarrow B^{\prime}$ is an embedding. Passing to a further subspace if necessary, we may assume that the nuclear norm of $K$ is strictly less than 1 . Let $N$ be a nuclear endomorphism of $B$ extending $K$ and having the same nuclear norm as $K$. Then, $\|N: B \rightarrow B\|<1$ and $\mathbf{1}_{B}-N$ is invertible, with $\left(\mathbf{1}_{B}-N\right)^{-1}=\sum_{k \geq 0} N^{k}$ with convergence in the operator norm. Now, it is easily seen that

$$
\left(\mathbf{1}_{B}-N\right) \circ P \circ\left(\mathbf{1}_{B}-N\right)^{-1}
$$

is a projection of $B$ (hence of $A$ ) onto $A^{\prime}$.

The proof also works replacing $\ell_{2}$ by any other "complementably minimal" space (those Banach spaces, all of whose infinite dimensional closed subspaces contain 
subspaces isomorphic to and complemented in the whole space) such as $\ell_{p}$ for $1<p<\infty$. This implies that Proposition 15 extends almost verbatim to $1<p<\infty$.

5.3. A twisted sum of $Z_{2}$ containing $\ell_{2}$ complemented. It is quite surprising that there exists a twisted sum of $Z_{2}$ containing complemented copies of $\ell_{2}$. But they do exist:

Proposition 16. There is a (nontrivial) exact sequence

$$
0 \longrightarrow Z_{2} \longrightarrow \ell_{2} \oplus \mathscr{X}^{(3)} \longrightarrow Z_{2} \longrightarrow 0 .
$$

Proof. Recall from [Castillo and Moreno 2004, p. 257] the construction of the so-called diagonal pushout sequence: in a commutative diagram

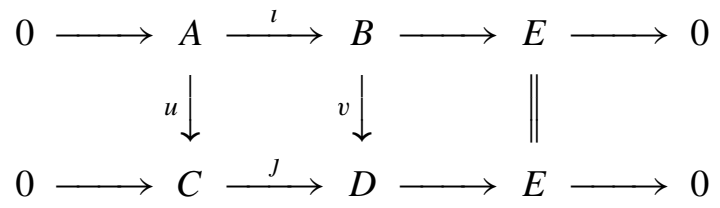

the following sequence is exact

$$
0 \longrightarrow A \stackrel{l \times u}{\longrightarrow} B \oplus C \stackrel{v \oplus-J}{\longrightarrow} D \longrightarrow 0,
$$

where $(l \times u)(a)=(l(a), u(a))$ and $(v \oplus-\jmath)(b, c)=v(b)-\jmath(c)$. Thus, taking $n=i=1$ and $k=j=2$ in $(6)$ for $\mathscr{F}=\mathscr{F}\left(\ell_{\infty}, \ell_{1}\right)$, one gets a commutative diagram

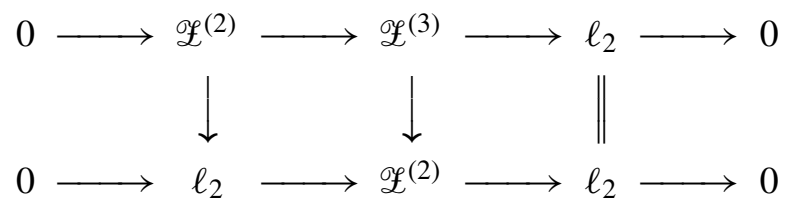

from which, recalling that $\mathscr{L}^{(2)}=Z_{2}$, one obtains an exact sequence

$$
0 \longrightarrow Z_{2} \longrightarrow \ell_{2} \oplus \mathscr{L}^{(3)} \longrightarrow Z_{2} \longrightarrow 0
$$

which is not trivial since, otherwise $Z_{2} \simeq Z_{2} \oplus Z_{2}=\ell_{2} \oplus \mathscr{L}^{(3)}$; this is impossible since $Z_{2}$ does not contain $\ell_{2}$ complemented.

Therefore $\ell_{2} \oplus \mathscr{L}^{(3)}$ is a twisted sum of $Z_{2}$, which contains complemented Hilbert subspaces. We cannot resist remarking that while nobody knows whether $Z_{2}$ is isomorphic to its hyperplanes, it is obvious that $\ell_{2} \oplus \mathscr{L}^{(3)}$ is isomorphic to its own hyperplanes.

\section{Open ends}

6.1. On the splitting of the first extension. Very little is known about the splitting of the "first" exact sequence $0 \rightarrow \mathscr{L}^{(1)} \rightarrow \mathscr{L}^{(2)} \rightarrow \mathscr{L}^{(1)} \rightarrow 0$ outside of the case in 
which it is induced by a couple of Banach lattices. On the other hand, Corollary 6 shows that once the first exact sequence obtained in an interpolation schema is nontrivial, the same happens to all the rest. Is the reciprocal true? That is, suppose that $\mathscr{L}^{(2)}$ is a trivial self-extension of $\mathscr{L}^{(1)}$. Does it follow that the extensions $0 \rightarrow \mathscr{L}^{(k)} \rightarrow \mathscr{L}^{(n+k)} \rightarrow \mathscr{L}^{(n)} \rightarrow 0$ are trivial for all values of $n$ and $k$ ?

6.2. Other twisted Hilbert spaces. Suppose we have a Banach space $X_{0}$ with a normalized basis that we use to consider $X_{0}$ inside $\ell_{\infty}$. Take $X_{1}=\overline{X_{0}^{\prime}}$ the complex conjugate of the closure $X_{0}^{\prime}$ of the subspace spanned by the coordinate functionals in $X_{0}^{*}$. Then $\left(X_{0}, X_{1}\right)$ is a Banach couple, $\left[X_{0}, X_{1}\right]_{1 / 2}$ is a Hilbert space [Pisier and $X u$ 2003, p. 1471], and thus $\mathscr{L}^{(2)}$ is a twisted Hilbert space. We believe that $\mathscr{L}^{(2)}$ is a Hilbert space if and only if $X_{0}=\ell_{2}$.

6.3. Other interpolation methods. It is the feeling of the authors that most of the work done in this paper could be reproduced for real interpolation by either the $K$ or $J$ methods with a careful analysis of the work done in [Carro et al. 1995]. It would be interesting to know to what extent the same occurs for other interpolation methods.

6.4. About the vanishing of $\mathbf{E x t}^{2}$. A problem on the horizon, for us, is whether the second derived functor Ext ${ }^{2}$ vanishes on Hilbert spaces, which can be understood as a twisted reading of a question of Palamodov for Fréchet spaces [1971, Section 12, Problem 6].

Given Banach spaces $A$ and $D$, one considers the set of all possible four-term exact sequences

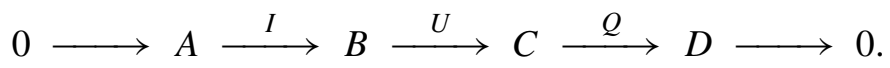

Under a certain equivalence relation, which is not necessary to define here, the set of such four-term exact sequences becomes a linear space denoted by $\operatorname{Ext}^{2}(D, A)$, whose zero is (the class of all exact sequences equivalent to)

$$
0 \longrightarrow A \rightleftharpoons D \stackrel{0}{\longrightarrow} D \rightleftharpoons D \longrightarrow
$$

It is important to realize that if we are given a short exact sequence of the form

$$
0 \longrightarrow A \stackrel{I}{\longrightarrow} B \stackrel{P}{\longrightarrow} E \longrightarrow 0
$$

and another sequence of the form

$$
0 \longrightarrow \mathrm{I} \stackrel{J}{\longrightarrow} C \stackrel{Q}{\longrightarrow} D \longrightarrow 0
$$

then we may form a four-term sequence

$$
0 \longrightarrow A \stackrel{I}{\longrightarrow} B \stackrel{U}{\longrightarrow} C \stackrel{Q}{\longrightarrow} D \longrightarrow 0
$$


just taking $U=J \circ P$. This resulting "long" sequence will be zero in $\operatorname{Ext}^{2}(D, A)$ if and only if (14) and (15) fit inside a commutative diagram

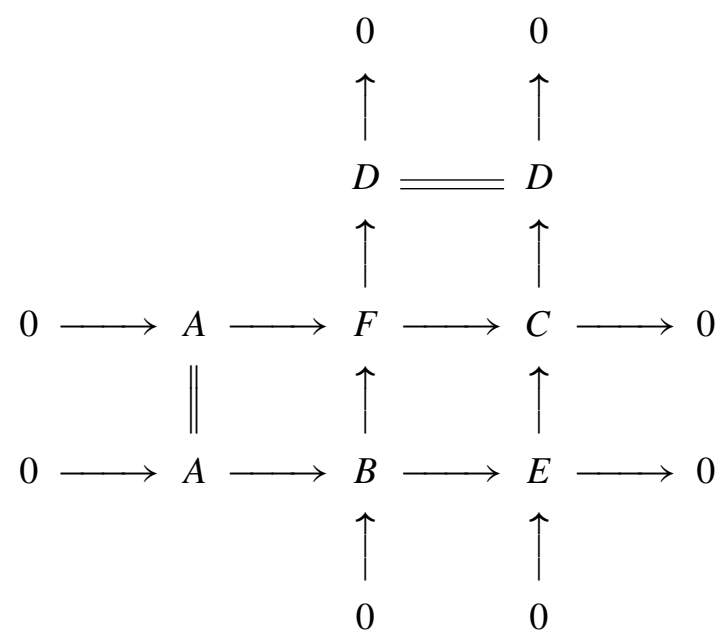

whose rows and columns are exact.

The skeptical reader will wonder how this is related to the main subject of the paper. Let $\mu$ be a $\sigma$-finite measure on a measure space $S$ and let $L_{0}$ be the space of all (complex) measurable functions on $S$, where we identify two functions if they agree almost everywhere. If $X$ is a Köthe space on $\mu$, then a centralizer on $X$ is a homogeneous mapping $\Omega: X \rightarrow L_{0}$ having the following property: there is a constant $C=C(\Omega)$ such that, for every $f \in X$ and every $a \in L_{\infty}$, the difference $\Omega(a f)-a \Omega(f)$ belongs to $X$ and

$$
\|\Omega(a f)-a \Omega(f)\|_{X} \leq C\|a\|_{\infty}\|f\|_{X} .
$$

Every centralizer is quasilinear, so it induces a twisted sum

$$
X \oplus_{\Omega} X=\{(y, x): x, y-\Omega(x) \in X\}
$$

which is quasinormed by the functional $\|(y, x)\|_{\Omega}=\|y-\Omega(x)\|_{X}+\|x\|_{X}$. A widely ignored result by Kalton states that if $X$ is superreflexive then one can construct an admissible space of analytic functions $\mathscr{F}$ on a disc centered at the origin such that:

- $X=X_{0}^{(1)}$ (evaluation at 0 ) up to equivalent norm;

- $\Omega \approx \Omega_{1,1}$, where $\Omega_{1,1}$ is the corresponding "derivation" (see Section 3.5).

This means that for every $x \in X$ the difference $\Omega(x)-\Omega_{1,1}(x)$ falls in $X$, and one has the estimate $\left\|\Omega(x)-\Omega_{1,1}(x)\right\|_{X} \leq K\|x\|_{X}$ for some constant $K$ and every $x \in X$. Actually one can construct $\mathscr{F}$ by using no more than three Köthe spaces on the boundary of the disc [Kalton 1992, Theorem 7.9]; if $\Omega$ is "real" in the sense that it takes real functions into real functions, then two Köthe spaces on a strip 
suffice [op. cit., Theorem 7.6]. In particular since $X \oplus_{\Omega} X=X \oplus_{\Omega_{1,1}} X=X_{0}^{(2)}$, up to equivalent (quasi-)norms, we see that the self-extension induced by $\Omega$ fits into the commutative diagram (the operators $l_{n, k}$ are those appearing in Proposition 2)

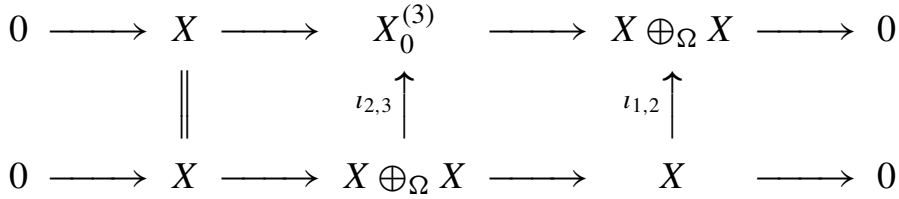

which, when completed, has the same form as (16), witnessing that the juxtaposition of two copies of the extension induced by $\Omega$, namely

$0 \longrightarrow X \longrightarrow X \oplus_{\Omega} X \longrightarrow X \oplus_{\Omega} X \longrightarrow X \longrightarrow 0$,

is zero in $\operatorname{Ext}^{2}(X, X)$. We do not know what happens with two different centralizers; more specifically, we ask the following: Let $\Omega$ and $\Phi$ be centralizers on a superreflexive Köthe space $X$ and consider the twisted sums $X \oplus_{\Omega} X$ and $X \oplus_{\Phi} X$. If, as before, we set $I(x)=(x, 0), U(x, y)=(y, 0)$, and $Q(x, y)=y$, can the exact sequence

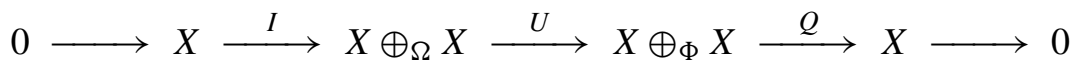

be nonzero in $\operatorname{Ext}^{2}(X, X)$ ?

\section{References}

[Cabello Sánchez 1999] F. Cabello Sánchez, “Twisted Hilbert spaces”, Bull. Austral. Math. Soc. 59:2 (1999), 177-180. MR 99m:46030 Zbl 0958.46003

[Cabello Sánchez and Castillo 2004] F. Cabello Sánchez and J. M. F. Castillo, "Uniform boundedness and twisted sums of Banach spaces", Houston J. Math. 30:2 (2004), 523-536. MR 2005f:46032 Zbl 1063.46012

[Cabello Sánchez et al. 2012] F. Cabello Sánchez, J. M. F. Castillo, and J. Suárez, "On strictly singular nonlinear centralizers", Nonlinear Anal. 75:7 (2012), 3313-3321. MR 2891170 Zbl 1248.46013

[Carro et al. 1995] M. J. Carro, J. Cerdà, and J. Soria, "Higher order commutators in interpolation theory", Math. Scand. 77:2 (1995), 301-319. MR 97d:46094 Zbl 0852.46058

[Castillo and González 1997] J. M. F. Castillo and M. González, Three-space problems in Banach space theory, Lecture Notes in Mathematics 1667, Springer, Berlin, 1997. MR 99a:46034 Zbl 0914.46015

[Castillo and Moreno 2004] J. M. F. Castillo and Y. Moreno, "On the Lindenstrauss-Rosenthal theorem”, Israel J. Math. 140 (2004), 253-270. MR 2005d:46044 Zbl 1063.46003

[Castillo and Moreno Salguero 2007] J. M. F. Castillo and Y. Moreno Salguero, "Singular and cosingular exact sequences of quasi-Banach spaces", Arch. Math. (Basel) 88:2 (2007), 123-132. MR 2008b:46004 Zbl 1116.46012

[Castillo et al. 2012] J. M. F. Castillo, M. Simoes, and J. Suárez de la Fuente, "On a question of Pełczyński about strictly singular operators”, Bull. Pol. Acad. Sci. Math. 60:1 (2012), 27-36. MR 2901385 Zbl 1244.46002 
[Cwikel et al. 1989] M. Cwikel, B. Jawerth, M. Milman, and R. Rochberg, "Differential estimates and commutators in interpolation theory", pp. 170-220 in Analysis at Urbana, II (Urbana, IL, 19861987), edited by E. Berkson and T. Peck, London Math. Soc. Lecture Note Ser. 138, Cambridge Univ. Press, 1989. MR 90k:46157 Zbl 0696.46050

[Hilton and Stammbach 1971] P. J. Hilton and U. Stammbach, A course in homological algebra, Graduate Texts in Mathematics 4, Springer, New York-Berlin, 1971. MR 49 \#10751 Zbl 0238.18006

[Kalton 1978] N. J. Kalton, "The three space problem for locally bounded $F$-spaces”, Compositio Math. 37:3 (1978), 243-276. MR 80j:46005 Zbl 0395.46003

[Kalton 1992] N. J. Kalton, "Differentials of complex interpolation processes for Köthe function spaces”, Trans. Amer. Math. Soc. 333:2 (1992), 479-529. MR 92m:46111 Zbl 0776.46033

[Kalton and Montgomery-Smith 2003] N. Kalton and S. Montgomery-Smith, "Interpolation of Banach spaces", pp. 1131-1175 in Handbook of the geometry of Banach spaces, II, edited by W. B. Johnson and J. Lindenstrauss, North-Holland, Amsterdam, 2003. MR 2006c:46061 Zbl 1041.46012

[Kalton and Peck 1979] N. J. Kalton and N. T. Peck, "Twisted sums of sequence spaces and the three space problem”, Trans. Amer. Math. Soc. 255 (1979), 1-30. MR 82g:46021 Zbl 0424.46004

[Kato 1958] T. Kato, "Perturbation theory for nullity, deficiency and other quantities of linear operators", J. Analyse Math. 6 (1958), 261-322. MR 21 \#6541 Zbl 0090.09003

[Palamodov 1971] V. P. Palamodov, "Homological methods in the theory of locally convex spaces", Uspehi Mat. Nauk 26:1(157) (1971), 3-65. In Russian; translated in Russ. Math. Surv. 26(1971): 1 (1974), 1-64. MR 45 \#2442 Zbl 0247.46070

[Pełczyński 1965] A. Pełczyński, "On strictly singular and strictly cosingular operators, I: Strictly singular and strictly cosingular operators in C(S)-spaces”, Bull. Acad. Polon. Sci. Sér. Sci. Math. Astronom. Phys. 13 (1965), 31-36. MR 31 \#1563 Zbl 0138.38604

[Pisier and $\mathrm{Xu}$ 2003] G. Pisier and Q. Xu, "Non-commutative $L^{p}$-spaces", pp. 1459-1517 in Handbook of the geometry of Banach spaces, II, edited by W. B. Johnson and J. Lindenstrauss, North-Holland, Amsterdam, 2003. MR 2004i:46095 Zbl 1046.46048

[Rochberg 1996] R. Rochberg, "Higher order estimates in complex interpolation theory", Pacific J. Math. 174:1 (1996), 247-267. MR 97i:46134 Zbl 0866.46047

[Rochberg 2007] R. Rochberg, "Uses of commutator theorems in analysis", pp. 277-295 in Interpolation theory and applications, edited by L. De Carli and M. Milman, Contemp. Math. 445, Amer. Math. Soc., Providence, RI, 2007. MR 2009b:46053 Zbl 1143.46306

[Rochberg and Weiss 1983] R. Rochberg and G. Weiss, "Derivatives of analytic families of Banach spaces”, Ann. of Math. (2) 118:2 (1983), 315-347. MR 86a:46099 Zbl 0539.46049

Received October 1, 2013.

FÉlix CABEllo SÁNCHEZ

Departamento de Matemáticas

UNIVERSIDAD DE EXTREMADURA

AVEnIDA DE ElVAS

06011 BADAJOZ

SPAIN

fcabello@unex.es 
Jesús M. F. CASTILlo

DEPARTAMENTO DE MATEMÁTICAS

UNIVERSIDAD DE EXTREMADURA

AVENIDA DE ELVAS

06011 BADAJOZ

SPAIN

castillo@unex.es

Nigel J. KALTON

DEPARTMENT OF MATHEMATICS

UNIVERSITY OF MISSOURI

COLUMBia, MO 65211

UNITED STATES

nigel@math.missouri.edu 


\title{
PACIFIC JOURNAL OF MATHEMATICS
}

\author{
msp.org/pjm
}

Founded in 1951 by E. F. Beckenbach (1906-1982) and F. Wolf (1904-1989)

\section{EDITORS}

Don Blasius (Managing Editor)

Department of Mathematics

University of California

Los Angeles, CA 90095-1555

blasius@math.ucla.edu

\author{
Paul Balmer \\ Department of Mathematics \\ University of California \\ Los Angeles, CA 90095-1555 \\ balmer@math.ucla.edu \\ Robert Finn \\ Department of Mathematics \\ Stanford University \\ Stanford, CA 94305-2125 \\ finn@math.stanford.edu \\ Sorin Popa \\ Department of Mathematics \\ University of California \\ Los Angeles, CA 90095-1555 \\ popa@math.ucla.edu
}

\author{
Vyjayanthi Chari \\ Department of Mathematics \\ University of California \\ Riverside, CA 92521-0135 \\ chari@math.ucr.edu \\ Kefeng Liu \\ Department of Mathematics \\ University of California \\ Los Angeles, CA 90095-1555 \\ liu@math.ucla.edu \\ Jie Qing \\ Department of Mathematics \\ University of California \\ Santa Cruz, CA 95064 \\ qing@ cats.ucsc.edu
}

\section{PRODUCTION}

Silvio Levy, Scientific Editor, production@msp.org

\section{SUPPORTING INSTITUTIONS}

ACADEMIA SINICA, TAIPEI

CALIFORNIA INST. OF TECHNOLOGY

INST. DE MATEMÁTICA PURA E APLICADA

KEIO UNIVERSITY

MATH. SCIENCES RESEARCH INSTITUTE

NEW MEXICO STATE UNIV.

OREGON STATE UNIV.

\author{
STANFORD UNIVERSITY \\ UNIV. OF BRITISH COLUMBIA \\ UNIV. OF CALIFORNIA, BERKELEY \\ UNIV. OF CALIFORNIA, DAVIS \\ UNIV. OF CALIFORNIA, LOS ANGELES \\ UNIV. OF CALIFORNIA, RIVERSIDE \\ UNIV. OF CALIFORNIA, SAN DIEGO \\ UNIV. OF CALIF., SANTA BARBARA
}

\author{
Daryl Cooper \\ Department of Mathematics \\ University of California \\ Santa Barbara, CA 93106-3080 \\ cooper@math.ucsb.edu \\ Jiang-Hua Lu \\ Department of Mathematics \\ The University of Hong Kong \\ Pokfulam Rd., Hong Kong \\ jhlu@maths.hku.hk \\ Paul Yang \\ Department of Mathematics \\ Princeton University \\ Princeton NJ 08544-1000 \\ yang@math.princeton.edu
}

These supporting institutions contribute to the cost of publication of this Journal, but they are not owners or publishers and have no responsibility for its contents or policies.

See inside back cover or msp.org/pjm for submission instructions.

The subscription price for 2015 is US \$420/year for the electronic version, and \$570/year for print and electronic.

Subscriptions, requests for back issues and changes of subscribers address should be sent to Pacific Journal of Mathematics, P.O. Box 4163, Berkeley, CA 94704-0163, U.S.A. The Pacific Journal of Mathematics is indexed by Mathematical Reviews, Zentralblatt MATH, PASCAL CNRS Index, Referativnyi Zhurnal, Current Mathematical Publications and Web of Knowledge (Science Citation Index).

The Pacific Journal of Mathematics (ISSN 0030-8730) at the University of California, c/o Department of Mathematics, 798 Evans Hall \#3840, Berkeley, CA 94720-3840, is published twelve times a year. Periodical rate postage paid at Berkeley, CA 94704, and additional mailing offices. POSTMASTER: send address changes to Pacific Journal of Mathematics, P.O. Box 4163, Berkeley, CA 94704-0163.

PJM peer review and production are managed by EditFLOW ${ }^{\circledR}$ from Mathematical Sciences Publishers.

\section{PUBLISHED BY}

\section{mathematical sciences publishers \\ nonprofit scientific publishing}

http://msp.org/

(C) 2015 Mathematical Sciences Publishers 


\section{PACIFIC JOURNAL OF MATHEMATICS}

Volume $276 \quad$ No. $2 \quad$ August 2015

Free evolution on algebras with two states, II

257

MiCHAEL ANSHELEVICH

Systems of parameters and holonomicity of A-hypergeometric systems

281

Christine Berkesch Zamaere, STEPHEN GRIFfeth and EZRA Miller

Complex interpolation and twisted twisted Hilbert spaces

287

FÉlix Cabello SÁnChez, Jesús M. F. Castillo and Nigel J. Kalton

The ramification group filtrations of certain function field extensions

309

JEFFREY A. CASTAÑEDA and QINGQUAN WU

A mean field type flow, II: Existence and convergence

JEAN-BAPTISTE CASTÉRAS

Isometric embedding of negatively curved complete surfaces in Lorentz-Minkowski space

BING-LONG CHEN and LE YIN

The complex Monge-Ampère equation on some compact Hermitian manifolds

JIANCHUN CHU

Topological and physical link theory are distinct

ALEXANDER COWARD and JOEL HASS

The measures of asymmetry for coproducts of convex bodies

Qi GuO, JinFEng GuO and XunLi Su

Regularity and analyticity of solutions in a direction for elliptic equations

YongYANG Jin, DONGSHENG LI and XU-JIA WANG

On the density theorem for the subdifferential of convex functions on Hadamard spaces

Mina Movahedi, Daryoush Behmardi and Seyedehsomayeh

HOSSEINI

$L^{p}$ regularity of weighted Szegó projections on the unit disc

SAMANGi MunASINGHE and Yunus E. ZEYTUnCU

Topology of complete Finsler manifolds admitting convex functions

SORIN V. SABAU and KatSUHIRO SHIOHAMA

Variations of the telescope conjecture and Bousfield lattices for localized categories of spectra

F. LUKE WOLCOTT 